11,17

\title{
Кинетика зарождения жидкой фазы в растянутом ГЦК-кристалле: молекулярно-динамическое моделирование
}

\author{
(C) В.Г. Байдаков, А.О. Типеев \\ Институт теплофизики УрО РАН, \\ Екатеринбург, Россия \\ E-mail: baidakov@itp.uran.ru
}

(Поступила в Редакцию 8 февраля 2018 г.)

Исследована кинетика спонтанного образования жидкой фазы в растянутом (перегретом) леннардджонсовском ГЦК-кристалле. В молекулярно-динамических экспериментах определены основные параметры процесса зародышеобразования: частота зародышеобразования $J$, коэффициент диффузии зародышей $D_{*}$, неравновесный фактор Зельдовича $Z$, размер критического зародыша $R_{*}$. Расчеты проведены при отрицательных - от конечной точки линии плавления, и положительных, до 8 раз превышающих критическое, давлениях. Результаты моделирования сопоставлены с классической теорией гомогенного зародышеобразования. Установлено, что теория качественно правильно воспроизводит динамику развития процесса. При хорошем количественном согласии теории и моделирования по скорости перехода зародыша жидкой фазы через критический размер имеет место большое расхождение по числу критических зародышей в единице объема метастабильной фазы. Для больших перегревов и отрицательных давлений вклад энергии упругих напряжений в движущую силу фазового превращения мал и в первом приближении им можно пренебречь. Рассогласование теории и результатов моделирования может быть устранено, если принять, что поверхностная свободная энергия искривленной межфазной границы кристалл-жидкая капля на 30-35\% меньше, чем плоской границы.

Исследование выполнено за счет гранта Российского научного фонда (проект 18-19-00276).

DOI: 10.21883/FTT.2018.09.46402.034

\section{1. Введение}

Фазовый переход первого рода предполагает наличие метастабильных состояний. Распад метастабильного состояния начинается с образования и последующего роста зародыша новой фазы. В идеальном кристалле, как в жидкости и паре, при отсутствии открытых поверхностей и инициирующих фазовый переход полей, зародыши возникают в результате термических флуктуаций (гомогенное зародышеобразование) [1]. Реальные кристаллы практически всегда содержат дефекты, такие как дислокации, структурные комплексы, создаваемые примесями и межузельными атомами, и т.п. Дефекты понижают высоту активационного барьера, отделяющего метастабильное состояние от стабильного, облегчая тем самым процесс фазового распада.

Гомогенное зародышеобразование определяет кинетическую границу предельной прочности твердого тела на разрыв. Растяжения, близкие к предельным, реализуются в ударно-волновых экспериментах [2,3]. Малые значения характерных временны́х масштабов и объемов, в которых создаются такие растяжения, позволяют надеяться на достижимость в этом случае спонтанного зародышеобразования.

Другой подход к изучению гомогенного зародышеобразования в твердом теле - компьютерный эксперимент. Методом молекулярной динамики (МД) предельные перегревы кристалла при давлениях, близких к атмосферному, исследовались в работах [4-6]. По данным $\mathrm{Lu}$ и $\mathrm{Li}$ [5] массовое образование зародышей жидкой фазы в леннард-джонсовском гранецентрированном кубическом (ГЦК) кристалле наблюдается при температуре $T_{n} \approx 1.2 T_{m}$, где $T_{m}$ - температура плавления. Для чистых металлов и простых соединений величина $T_{n}$ варьируется в пределах $(1.08-1.43) T_{m}$ и слабо зависит от скорости нагрева [6]. В широком интервале давлений, включая отрицательные, информация о предельных перегревах кристаллической фазы получена в работах [7-10].

Проявляя неустойчивость к процессу зарождения новой фазы, метастабильная система сохраняет восстановительную реакцию на бесконечно малые возмущения. В работах $[8,10]$ по данным МД-моделирования из условия $(\partial p / \partial \rho)_{T}=0$ определена граница устойчивости леннард-джонсовского ГЦК-кристалла относительно однородных объемных деформаций - спинодаль. Соответствующие этой границе значения растягивающих напряжений известны как „идеальная“ или „теоретическая“ прочность кристалла.

В данной работе методом МД-моделирования исследуется кинетика фазового распада в растянутом леннардджонсовском ГЦК-кристалле.

Полученные данные интерпретируются в рамках классической теории гомогенного зародышеобразования (КТГЗ).

Статья построена следующим образом. В разд. 2 изложены основы КТГЗ применительно к фазовому переходу твердое тело-жидкость. В разд. 3 описаны детали моде- 
лирования. Результатам расчетов и их сопоставлению с КТГЗ посвящен разд. 4. Раздел 5 - заключение.

\section{2. Теория гомогенного зародышеобразования}

При постоянстве температуры $T_{0}$ и давления $p_{0}$ минимальная работа образования зародыша жидкой фазы в изотропной упругой среде определяется суммой поверхностной, объемной, молекулярной и деформационной работ [1]:

$$
W=\gamma A+\left(p_{0}-p_{l}\right) V_{l}+\left(\mu_{l}-\mu_{0}\right) N_{l}+e V_{l},
$$

где $A, V_{l}$ и $N_{l}$ - площадь поверхности, объем и число частиц зародыша, $\gamma$ - эффективная поверхностная свободная энергия границы раздела твердое тело-жидкость, и $p_{l}$ и $\mu_{l}$ - давление и химический потенциал в зародыше, $e$ - плотность упругой энергии системы зародыш-твердое тело.

Упругая энергия, как и поверхностная, при заданных $T_{0}, p_{0}$ и $V_{l}$ зависит от формы зародыша и в общем случае должна быть определена из условия минимума $W$. В отличие от флюидной фазы, сферическая форма зародыша в упругой среде не всегда является энергетически наиболее выгодной $[11,12]$. Она оптимальна при больших перегревах (растяжениях) [12], но при малой степени метастабильности приводит к нефизическому решению (упругому термодинамическому гистерезису [13]). В данной работе рассматриваются перегревы (растяжения), близкие к спинодальным. Результаты МД-моделирования процесса образования жидкой фазы в леннардджонсовском ГЦК-кристалле свидетельствуют, что при больших перегревах зародыши имеют форму, близкую к сферической [14].

Возникновение зародыша жидкой фазы в твердом теле сопровождается его деформациями. Расчет упругой энергии связан с определением полей деформаций и напряжений. В качестве исходного состояния обычно выбирается недеформированное твердое тело $(p=0)$, которое при определенных температурах может оказаться термодинамически неустойчивым. Как показано в работах $[12,13]$, для удельной упругой энергии внутренних напряжений, возникающих при контакте двух фаз, различающихся собственной деформацией, можно записать

$$
e=\frac{2 K_{l} \mu_{s}}{3 K_{l}+4 \mu_{s}}\left(\frac{\Delta \rho_{0}}{\rho_{s 0}}+\frac{K_{s}-K_{l}}{K_{s} K_{l}} p_{0}\right)^{2} .
$$

Здесь $\mu_{s}$ - модуль сдвига твердого тела, $K_{s}, K_{l}$ и $\Delta \rho_{0}=\rho_{s 0}-\rho_{l 0}-$ модули объемного сжатия и разность плотностей твердой и жидкой фаз. Зародыш жидкой фазы считается сферическим. Величины, входящие в формулу (2), берутся при $p=0$ и температуре $T_{0}$.

Учитывая малую сжимаемость жидкой и твердой фаз, разность химических потенциалов в предпослед- нем члене (1) можно представить в виде $\mu_{l}-\mu_{0}=$ $=\vartheta_{l m}\left(p_{l}-p_{m}\right)-\vartheta_{s m}\left(p_{0}-p_{m}\right)$, где $p_{m}-$ давление, а $\vartheta_{l m}=\rho_{l m}^{-1}, \vartheta_{s m}=\rho_{s m}^{-1}-$ удельные объемы жидкости и кристалла на линии плавления при температуре $T_{0}$. После подстановки этого соотношения в (1) получаем

$$
W=\gamma A+\left[\left(p_{0}-p_{m}\right) \Delta \rho_{m} / \rho_{s m}+e\right] V_{l} .
$$

Критический зародыш находится в неустойчивом равновесии с окружающей средой. Из условия равновесия и (3) для радиуса критического зародыша имеем

$$
R_{*}=\frac{-2 \gamma}{\left(p_{0}-p_{m}\right) \frac{\Delta \rho_{m}}{\rho_{s m}}+e} .
$$

Подстановка (4) в (3) дает работу образования критического зародыша

$$
W_{*}=\frac{16 \pi \gamma^{3}}{3\left[\left(p_{0}-p_{m}\right) \frac{\Delta \rho_{m}}{\rho_{s m}}+e\right]^{2}} .
$$

Определяющей стадией нуклеации является прохождение зародышем вершины активационного барьера $W$. В КТГЗ движение зародыша через активационный барьер трактуется как процесс диффузии по оси его размера [15]. Задача определения частоты зародышеобразования - числа жизнеспособных зародышей новой фазы, образующихся в единице объема метастабильной системы за единицу времени, помимо определения величины активационного барьера, требует нахождения коэффициента диффузии зародышей.

Коэффициент диффузии на вершине активационного барьера определяется через макроскопическую скорость его роста $d R / d t$ из условия отсутствия потока зародышей вдоль оси размера $[16]$ :

$$
\frac{d R}{d t}=-\frac{D_{*}}{k_{\mathrm{B}} T_{0}} \frac{d W}{d R},
$$

где $k_{\mathrm{B}}-$ постоянная Больцмана.

Для нахождения $d R / d t$ нужно принять модель определяющего процесса вблизи растущего зародыша. Будем полагать, что фактором, сдерживающим рост жидкой капли в упругой среде, является кинетика обмена частиц между твердой и жидкой фазами. По аналогии с теорией кристаллизации [17] считаем механизм плавления (роста жидкой капли) нормальным. В этом случае для скорости движения границы раздела фаз можно записать

$$
\frac{d R}{d t}=\kappa\left\{\left[p_{l}\left(T_{0}\right)-p\right]-\frac{2 \gamma}{R}\right\},
$$

где $\kappa$ - кинетический коэффициент, характеризующий частоту обмена частицами между твердой и жидкой фазами.

Линеаризуя уравнение (7) и производную $d W / d R$ вблизи вершины активационного барьера для коэффициента диффузии зародышей вдоль оси $R$ из (6), имеем

$$
D_{*}=\frac{3 \kappa k_{\mathrm{B}} T_{0}}{2 \pi R_{*}^{2}} .
$$


Зависимость $\kappa$ от термодинамических параметров состояния найдем, воспользовавшись теорией абсолютных скоростей реакций [18]. Аналогично тому, как это сделано для зародыша кристаллической фазы в переохлажденной жидкости [19], можно записать

$$
\kappa=\frac{v_{0}}{k_{\mathrm{B}} T_{0} \rho_{0}^{4 / 3}} \exp \left(-U / k_{\mathrm{B}} T_{0}\right),
$$

где $v_{0}$ - частота колебаний частиц на поверхности зародыша, $U$ - энергия активации, которая принимается равной теплоте плавления в расчете на одну частицу. Величину $v_{0}$ можно оценить из соотношения $h v_{0} \approx k_{\mathrm{B}} T_{0}$, где $h$ - постоянная Планка.

Частота зародышеобразования - конечный результат стационарной КТГЗ, определяется выражением [15]:

$$
J=C Z D_{*} \exp \left(-W_{*} / k_{\mathrm{B}} T_{0}\right)=J_{0} \exp \left(-W_{*} / k_{\mathrm{B}} T_{0}\right),
$$

где $C$ - нормировочный множитель равновесной функции распределения зародышей по размерам, $Z$ - неравновесный фактор Зельдовича.

Величина $C$ пропорциональна плотности числа частиц в твердой фазе $\rho_{0}$ и производной $d N_{l} / d R$, т. е.

$$
\left.C \approx \rho_{0} \frac{d N_{l}}{d R}\right|_{*}=4 \pi R_{*}^{2} \rho_{0} \rho_{l} \approx 4 \pi R_{*}^{2} \rho_{0}^{2} .
$$

Неравновесный фактор Зельдовича определяет кривизну потенциального барьера в его вершине и равен

$$
Z=\left[-\left(\frac{d^{2} W}{d R^{2}}\right)_{*} \frac{1}{2 \pi k_{\mathrm{B}} T_{0}}\right]^{1 / 2}=2\left(\frac{\gamma}{k_{\mathrm{B}} T_{0}}\right)^{1 / 2}
$$

Таким образом, из (8), (11) и (12) для предэкспоненциального множителя в (10) имеем

$$
J_{0}=12 \kappa \rho_{0}^{2}\left(\gamma k_{\mathrm{B}} T_{0}\right)^{1 / 2} .
$$

Эта формула подобна аналогичному выражению для $J_{0}$, используемому в теории кристаллизации переохлажденной жидкости [19].

Альтернативой КТГЗ является теория переходного состояния, предполагающая инерционное движение зародыша через активационный барьер. Данная теория дает оценку сверху для величины предэкспоненциального множителя и согласно [20]:

$$
J_{0}=\rho_{0}\left(\frac{2 \rho_{0} R_{*} k_{\mathrm{B}} T_{0}}{m}\right)^{1 / 2},
$$

где $m$ - масса молекулы. В отсутствие трения инерционное движение зародыша приводит к независимости потока через барьер от формы вершины барьера.

\section{3. Молекулярно-динамическое моделирование}

Фазовый распад кристаллического состояния исследовался в системе леннард-джонсовских (ЛД) частиц.
Частицы размещались в узлах гранецентрированной кубической решетки. Число частиц в системе $N$ варьировалось от 2048 до 500000 . Радиус обрезания потенциала взаимодействия составлял $6.58 \sigma$, где $\sigma-$ параметр ЛД-потенциала. На границы системы, которая имела форму куба, налагались периодические граничные условия. Расчет модулей упругости проводился в $N V T$-ансамбле, фазовый распад протекал в $N V E$ условиях. Температура в $N V T$ ансамбле контролировалась с помощью термостата Нозе-Хувера [21]. При интегрировании уравнений движения частиц использовался ускоренный алгоритм Верле [22]. Шаг интегрирования по времени $\Delta t$ составлял $0.002 \tau_{L J}$ ЛД-единиц (ЛД единица времени $\tau_{L J}=\left(m \sigma^{2} / \varepsilon\right)^{1 / 2}$, где $\varepsilon-$ параметр потенциала). Далее все рассчитываемые величины приводятся в безразмерном виде и отмечаются верхним индексом (*). Единицами приведения выступают параметры потенциала ЛД $\sigma$ и $\varepsilon$, масса частицы $m$ и постоянная Больцмана $k_{\mathrm{B}}$. Приведенные температура $T^{*}=k_{\mathrm{B}} T / \varepsilon$, давление $p^{*}=p \sigma^{3} / \varepsilon$, плотность $\rho^{*}=\rho \sigma^{3}$, частота зародышеобразования $J^{*}=J \sigma^{4}(m / \varepsilon)^{1 / 2}$. Методика МД-расчета термодинамических величин подробно описана в $[8,14]$.

Появление в однородной метастабильной системе зародыша новой фазы - случайное событие. Для описания потока таких событий в эксперименте необходимо определить функцию распределения и ее первые моменты. При заданных $p_{0}, T_{0}$ время ожидания первого критического зародыша распределено по показательному закону Пуассона [23]. Среднее время ожидания жизнеспособного зародыша связано с частотой зародышеобразования соотношением $\bar{\tau}=(J V)^{-1}$, где $V$ - объем кристалла.

При МД-моделировании, после перевода системы в заданное метастабильное состояние $\left(p_{0}, T_{0}=\mathrm{const}\right)$, определялось время ожидания до начала фазового распада $\tau_{m}$. Момент разрушения метастабильной фазы фиксировался по изменениям давления и температуры системы, вызванных зародышеобразованием. Время $\tau_{m}$ есть сумма времен ожидания первого жизнеспособного зародыша $\tau$ и его последующего роста $\tau_{p}$ до момента фиксации фазового распада. Моделирование показало [14], что при больших степенях метастабильности фазовый распад инициируется одним зародышем, а время $\tau_{p} \ll \tau$. Поэтому принималось, что $\tau \approx \tau_{m}$.

\section{4. Результаты}

4.1. Частота зародыше образования. Кинетика зарождения жидкой фазы в леннард-джонсовском ГЦК-кристалле исследована при температурах $T_{0}^{*}=0.55,0.7,0.85,1.0$. Температура тройной точки $T_{t}^{*}=0.692$ [8]. Заход в метастабильную область осуществлялся путем изотермического растяжения кристалла. Первоначально кристалл переводился в некоторое промежуточное слабометастабильное состояние 
$\left(p_{0}<p_{+}<p_{m}, T_{0}\right)$, в котором его время жизни было более чем $10^{6}$ МД-шагов и строился ансамбль начальных конфигураций частиц. Для этого на фазовой траектории через каждые 20000 МД-шагов записывались координаты и скорости частиц в ячейке. Выделенные микроскопические состояния принимались за начальные. Из каждого такого начального состояния путем линейного масштабирования координат всех частиц в ячейке (уменьшением плотности системы) осуществлялся переход к исследуемому состоянию $\left(p_{0}, T_{0}\right)$. С целью сокращения времени релаксации и сохранения начальной температуры $T_{0}$ в течение первых 5000 МД-шагов после перехода производилась коррекция температуры.

Максимальное время ожидания появления первого закритического зародыша составляло около $10^{7}$ МД-шагов или $0.05 \mu \mathrm{s}$. В процессе фазового распада по 100 МД-шагам производилось усреднение полученных значений давления, температуры и внутренней энергии.

Образование в кристалле закритического зародыша жидкой фазы приводило к резкому росту давления и понижению температуры (рис. 1). За время жизни метастабильного состояния принималось время от перехода к состоянию $\left(p_{0}, T_{0}\right)$ до момента понижения температуры кристалла на $0.01 T_{0}$.

В каждом из исследуемых состояний $\left(p_{0}, T_{0}\right)$ регистрировалось от $n=50$ до 200 независимых событий плавления и определялось среднее время жизни $\bar{\tau}=\sum \tau / n$. В системах, содержащих разное число частиц, величина $\bar{\tau}$ была обратна пропорциональной объему системы: $\bar{\tau} \sim 1 / V$. Это свидетельствует об объемном характере зародышеобразования и отсутствии влияния периодических граничных условий.

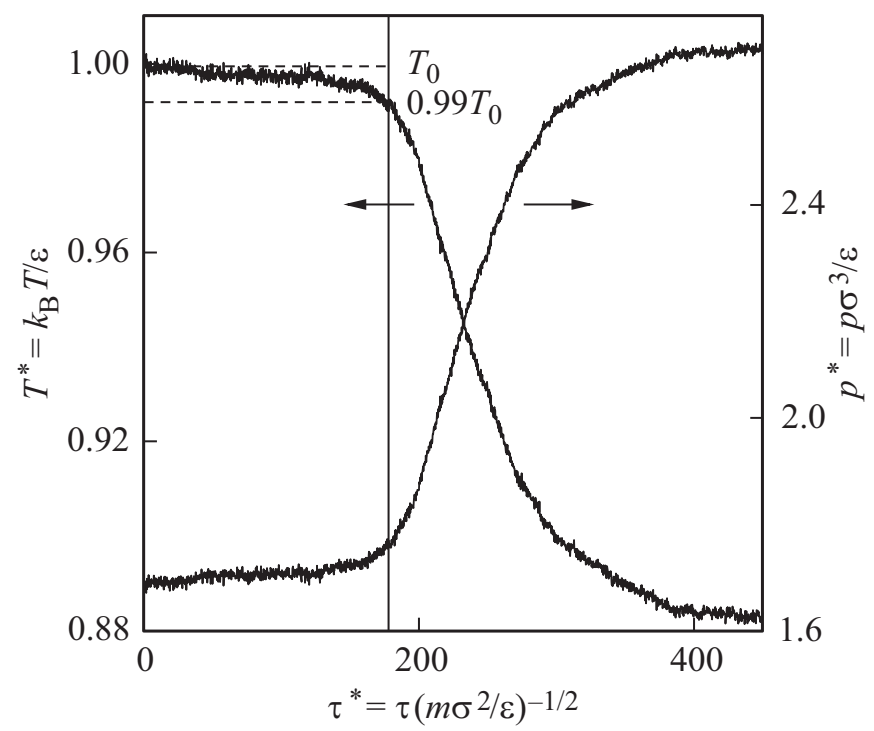

Рис. 1. Зависимость температуры и давления от времени при появлении зародыша жидкой фазы: $T_{0}^{*}=1.0, \rho_{0}^{*}=0.930$, $n=108000$. Вертикальной линией отмечено время начала фазового распада $\tau^{*}=178$.

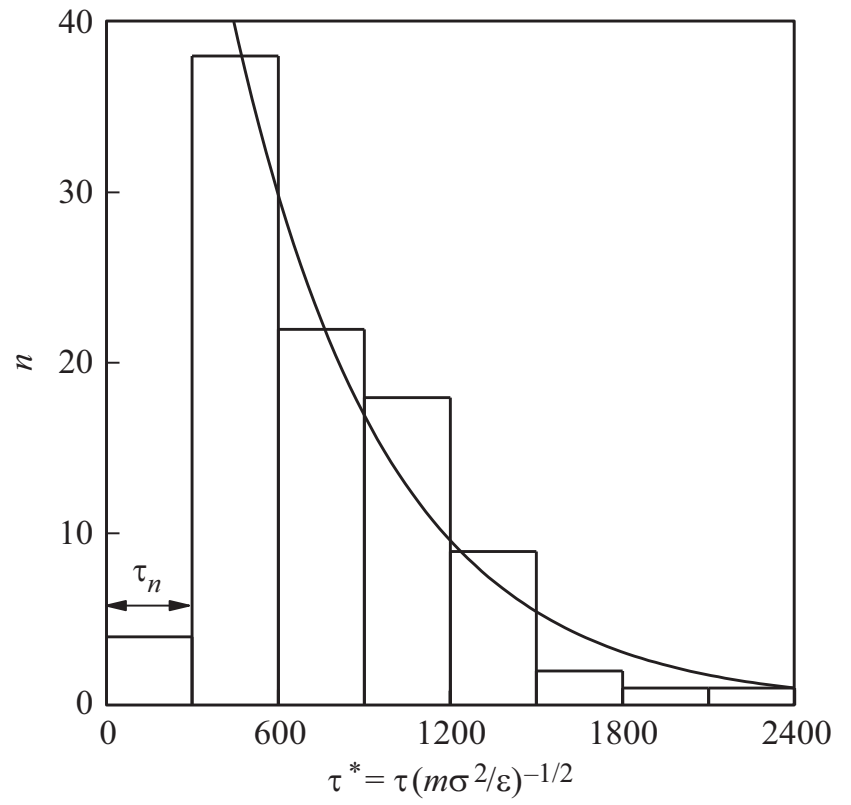

Рис. 2. Гистограмма распределения событий плавления ЛД-кристалла: $T_{0}^{*}=1.0, \quad \rho_{0}^{*}=0.935, \quad n=100, \quad \bar{\tau}^{*}=805$, $N=108000$. Плавная кривая - пуассоновское распределение с учетом времени запаздывания $\tau_{n}$.

При $T_{0}^{*}=0.55$ падение температуры в начале плавления затем сменялось ее резким ростом. Последнее связано с образованием кавитационной полости внутри закритического зародыша жидкой фазы. Основанный на использовании параметра центросимметрии анализ формы образующихся зародышей показал, что она близка к сферической [14].

На рис. 2 представлено распределение событий плавления $n$ по времени $\tau$. При малых $\tau$ имеет место отклонение от пуассоновского распределения (плавная кривая), связанное с установлением заданного термодинамического состояния $\left(p_{0}, T_{0}\right)$.

По данным о среднем времени ожидания плавления $\bar{\tau}$ определена частота зародышеобразования. Значения логарифма частоты зародышеобразования $J$ как функции плотности представлены на рис. 3. Погрешность определения $J$ сопоставима с размером точек рисунка. В приведенных единицах минимальное и максимальное значения $J$ составили соответственно $10^{-9}$ и $10^{-5}$, в размерных $-10^{31}$ и $10^{35} \mathrm{~s}^{-1} \mathrm{~m}^{-3}$.

Продвижение в область высоких частот зародышеобразования $J^{*}>10^{-5}\left(10^{35} \mathrm{~s}^{-1} \mathrm{~m}^{-3}\right.$, большие перегревы) ограничено условием стационарности процесса и числом частиц в модели. Принятое значение радиуса обрезания потенциала $r_{c}=6.58 \sigma$ при $N \leq 2048$ и $\rho_{0}^{*}>0.9$ из-за периодических граничных условий приводит к появлению нефизических взаимодействий. Уменьшение радиуса обрезания потенциала позволит сократить допустимое число частиц в системе, но приведет к изменению ее термодинамического состояния $\left(p_{0}, T_{0}\right)$. При $T_{0}^{*}=0.85$, 1.0 и $\rho_{0}^{*} \approx 0.90-0.94$ минимальное число частиц в 


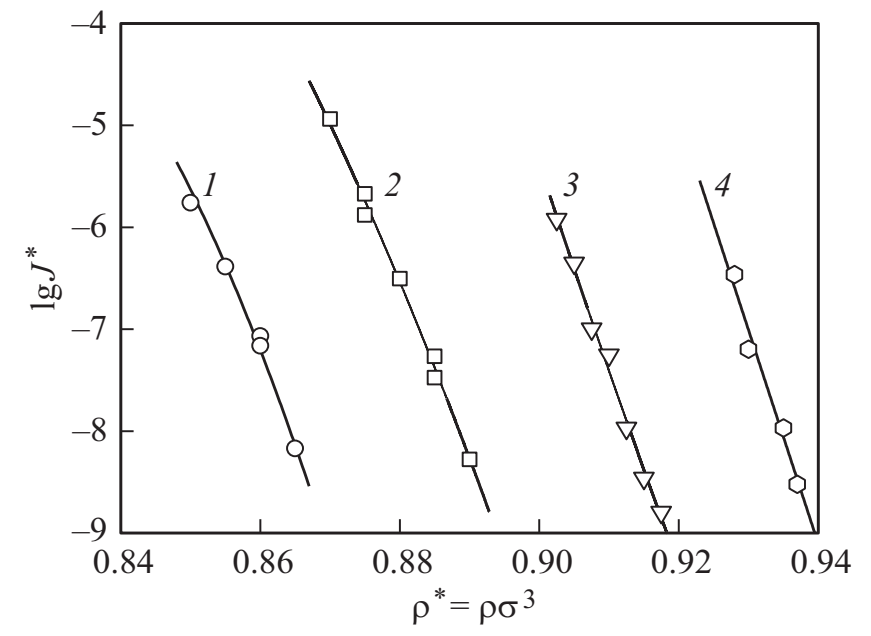

Рис. 3. Логарифм частоты зародышеобразования в перегретом ЛД-кристалле как функция плотности: $1-T_{0}^{*}=0.55,2-0.7$, $3-0.85,4-1.0$.

системе составило $N=8788$. Расчет частоты зародышеобразования в области меньших перегревов, которым отвечает $J^{*}<10^{-9}\left(10^{31} \mathrm{~s}^{-1} \mathrm{~m}^{-3}\right)$, связан с существенным ростом затрат компьютерного времени.

4.2. К ритический зародыш, неравновесный фактор Зельдовича и коэффициент диффузи и з ар одышей. При МД-моделировании независимым образом может быть определен каждый из входящих в предэкспоненциальный множитель $J_{0}=C Z D_{*}$ параметров.

Число частиц в критическом зародыше $N_{*}$ и неравновесный фактор Зельдовича $Z$ рассчитаны методом среднего времени первого перехода [24]. Для этого в каждом акте флуктуационнного плавления ЛД-кристалла через каждые 1000 МД-шагов сохранялись координаты всех частиц в ячейке. Затем с помощью алгоритма $q_{6}$ [25] в сохраненных конфигурациях производился поиск кристаллоподобных частиц (частиц, которые могут быть отнесены к кристаллической фазе). Параметры алгоритма $q_{6}$ были выбраны такими же, как при поиске кристаллических структур в переохлажденной жидкости [26]. В каждой конфигурации число частиц, принадлежащих жидкой фазе $N_{l}$, определялось как разность общего числа частиц в системе $N$ и числа частиц, принадлежащих кристаллической фазе $N_{s}$. Величина $N_{s}$ далее использовалась в качестве параметра порядка. Во всех термодинамических состояниях времена ожидания появления первого зародыша жидкой фазы, содержащего $N_{l}$ частиц, усреднялись по 30 событиям плавления. Средние значения времен ожидания зародыша $\langle t\rangle$ с $N_{l}$ частицами аппроксимировались функцией

$$
\left\langle t\left(N_{l}\right)\right\rangle=\frac{\bar{\tau}}{2}\left\{1-\operatorname{erf}\left[\pi^{1 / 2} Z_{*}\left(N_{l}-N_{*}\right)\right]\right\},
$$

где $\operatorname{erf}(x)$ - функция ошибок.
Результаты расчета $\left\langle t\left(N_{l}\right)\right\rangle$ представлены на рис. 4. Аппроксимация зависимости $\langle t\rangle$ от $N_{l}$ уравнением (15) (сплошная линия) дает $Z=(5 \pm 1) \cdot 10^{-3}, N_{*}=295 \pm 5$ $\left(T_{0}^{*}=0.7, \rho_{0}^{*}=0.885\right)$ и $Z=(3 \pm 1) \cdot 10^{-3}, N_{*}=494 \pm 4$ $\left(T_{0}^{*}=1.0, \rho_{0}^{*}=0.930\right)$.

Коэффициент диффузии зародышей вблизи критического размера рассчитывался как [27]:

$$
D_{*}=\frac{1}{2} \frac{\left\langle\Delta N_{l}^{2}(\tau)\right\rangle}{\tau},
$$

где $\Delta N_{l}(\tau)=\left[N_{l}(\tau)-N_{l}(0)\right]-$ изменение числа частиц в зародыше жидкой фазы за время $\tau$.

Для определения величины $D_{*}$ использовались конфигурации частиц, содержащие околокритические зародыши и сохраненные в алгоритме $q_{6}$ при расчетах $N_{*}$ и $Z$. В каждом состоянии отбиралось 5 случайных конфигураций с $N_{l}$, близких к $N_{*}: N_{l}=N_{*} \pm 20$, которые выступали как стартовые для новых МД-конфигураций с измененными значениями начальных скоростей частиц. Начало отсчета времени переопределялось каждый раз, когда $N_{l}$ было равно $N_{*}$. Расчеты прекращались при $N_{l}<100$ либо при $N_{l}>2 N_{*}$.

При $T_{0}^{*}=0.7$ и $\rho_{0}^{*}=0.885$ получено $D_{*}^{*}=48 \pm 10$; при $T_{0}^{*}=1.0$ и $\rho_{0}^{*}=0.930$ величина $D_{*}^{*}=142 \pm 56$. Большая погрешность в определении $D_{*}$ при $T_{0}^{*}=1.0$ связана со значительными флуктуациями числа частиц в зародыше жидкой фазы. Для более точного определения коэффициента диффузии зародышей необходимо существенно увеличить частоту запусков алгоритма расчета.

Таким образом, при прямом МД-моделировании для предэкспоненциального множителя получено

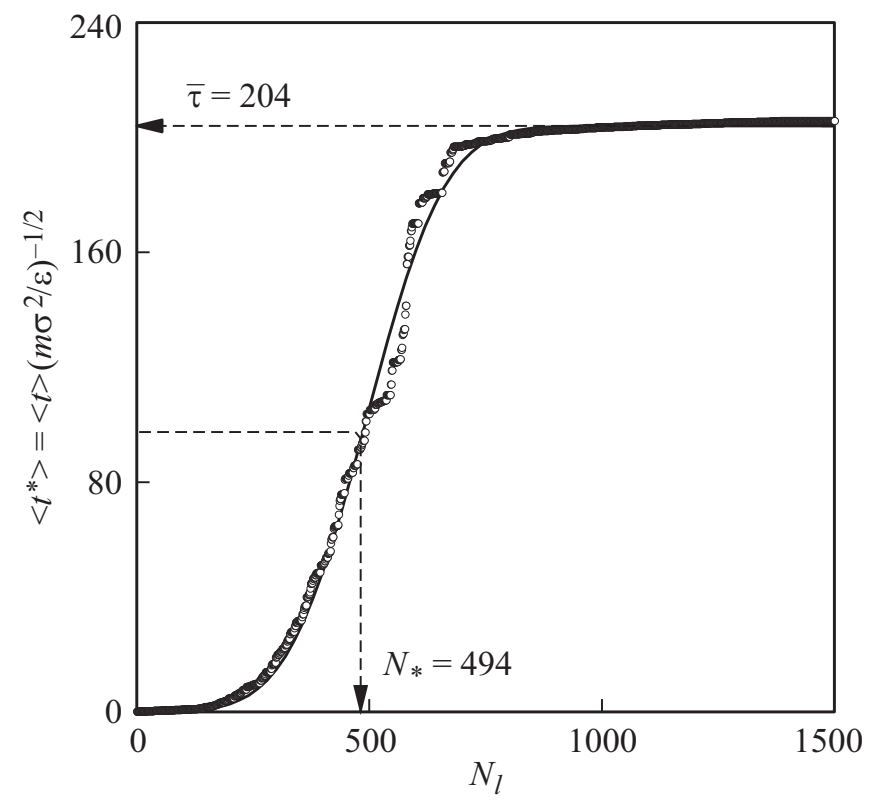

Рис. 4. Время ожидания появления в кристалле зародыша жидкой фазы определенного размера как функция числа содержащихся в нем частиц: $T_{0}^{*}=1.0, \rho_{0}^{*}=0.930, \bar{\tau}^{*}=204$, $N_{*}=494, N=32000$. Сплошная линия - расчет по уравнению (15). 
$J_{0}^{*}=0.21 \pm 0.05\left(T_{0}^{*}=0.7, \rho_{0}^{*}=0.885\right)$ и $J_{0}^{*}=0.40 \pm 0.16$ $\left(T_{0}^{*}=1.0, \rho_{0}^{*}=0.930\right)$.

4.3. Сопоставление результатов моделирования и КТГЗ. При сопоставлении результатов МД-моделирования и КТГЗ величина поверхностной свободной энергии на границе кристалл-жидкая капля принималась равной ее значению на плоской межфазной границе кристалл-жидкость [28]. Параметры линии плавления леннард-джонсовского кристалла $p_{m}, \rho_{s m}, \rho_{l m}$ и значения $\rho_{s 0}, \rho_{l 0}$ на нулевой изобаре определены в работах $[8,29]$.

Для бездефектного ЛД ГЦК-кристалла, находящегося под гидростатическим давлением, модули сдвига $\mu_{s}$ и объемного сжатия $K_{s}$ рассчитаны в работе [30]. Модуль объемного сжатия жидкой фазы $K_{l}=\rho(\partial p / \partial \rho)_{T}$ найден из уравнения состояния ЛД-флюида [31]. Упругая энергия $e$ определена из уравнения (2) по данным МД-моделирования. При изотермическом увеличении объема кристалла на $1-2 \%$ величина $e$ понижается на $6-19 \%$. Вклад упругой энергии в движущую силу процесса фазового распада $\left(\mu_{l}-\mu_{0}\right)$ убывает с понижением температуры. Он составляет $30-36 \%$ при $T_{0}^{*}=1.0$ и $4-5 \%$ при $T_{0}^{*}=0.55$.

Согласно уравнению (4), в исследованном диапазоне параметров состояния радиусы критических зародышей жидкой фазы $R_{*}^{*}=4.5-9.2$. Меньшее значение $R_{*}$ соответствует более низкой температуре. На изотерме величина $R_{*}$ уменьшается с ростом растяжения. Число частиц в критическом зародыше при $T_{0}^{*}=0.7$ и $\rho_{0}^{*}=0.885$ составляет $N_{*}=910$, а при $T_{0}^{*}=1.0$ и $\rho_{0}^{*}=0.930$ величина $N_{*}=2060$. Эти значения примерно в $3-4$ раза больше полученных при МД-моделировании методом среднего времени первого перехода (см. разд. 4.2). Пренебрежение упругой энергией в уравнении (4) уменьшает величину рассогласования с данными МД-моделирования до $1.3-1.7$ раз.

Рассчитанные с учетом упругой энергии по уравнениям (13) и (14) значения предэкспоненциального множителя $J_{0}$ в выражении для частоты зародышеобразования (10) составляют $J_{0}^{*}=2.6-9.5$ и $J_{0}^{*}=1.7-3.9$ соответственно. Пренебрежение упругой энергией приводит к повышению $J_{0}$ на $(5-10) \%$.

Результаты прямого МД-расчета (см. разд. 4.2) отличаются от данных уравнений (13), (14) примерно на порядок. Ввиду очень сильной зависимости частоты зародышеобразования от давления (плотности) указанные различия в $J_{0}$ незначительно сказываются на величине предельного растяжения кристалла.

Учет упругих напряжений повышает устойчивость метастабильного кристалла по отношению к фазовому распаду. Образование критического зародыша жидкой фазы в этом случае требует большей затраты работы. Рассчитанная по уравнению (3) приведенная величина активационного барьера $W_{*} / k_{\mathrm{B}} T_{0}$ (число Гиббса) с повышением температуры возрастает от $52-57\left(T_{0}^{*}=0.55\right)$ до 147-204 $\left(T_{0}^{*}=1.0\right)$. Пренебрежение упругой энергией дает $W_{*} / k_{\mathrm{B}} T_{0}=48-83$. В исследованных системах

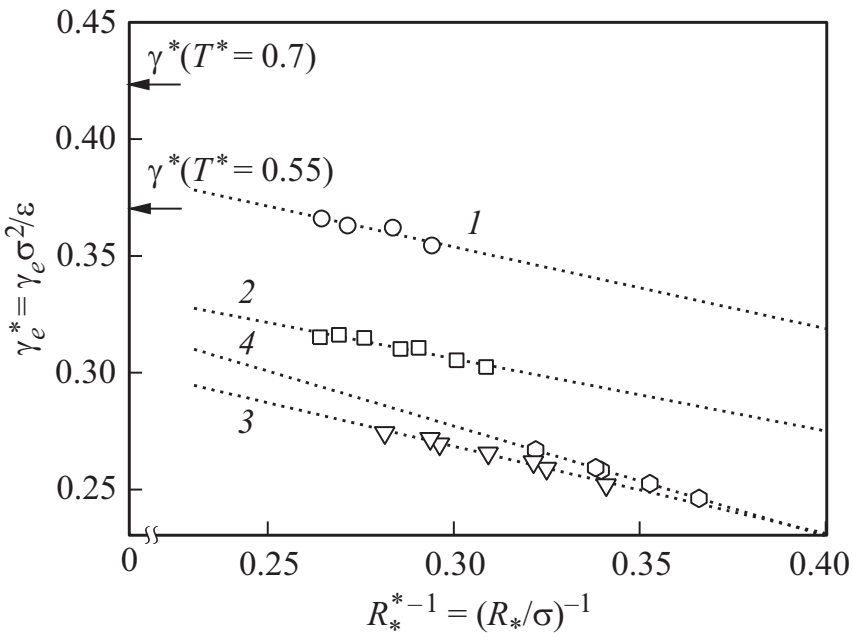

Рис. 5. Зависимость эффективной поверхностной свободной энергии на межфазной границе кристалл-зародыш жидкой фазы от кривизны поверхности натяжения. $1-T_{0}^{*}=1.0$ $\left(\gamma^{*}=0.569\right), 2-0.85$ (0.491), $3-0.7$ (0.424), $4-0.55$ $(0.369)$. Стрелками отмечены значения $\gamma$ на плоской межфазной границе кристалл-жидкость.

из $N=32000-500000$ частиц столь высокие активационные барьеры не могут быть преодолены за характерные времена МД-экспериментов (10 $0^{7}$ МД-шагов). При прямом МД-моделировании процессов кипения, конденсации и кристаллизации величина $W_{*} / k_{\mathrm{B}} T_{0}$ обычно не превышает 20-25 [32-34].

Таким образом, КТГЗ дает для $J$ значения на 16-70 порядков ниже полученных при МД-моделировании методом среднего времени жизни. Отклонения тем больше, чем выше температура. Если пренебречь упругой энергией в работе образования критического зародыша (5), то рассогласование по $J$ составит 14-27 порядков.

Расхождения КТГЗ и результатов МД-моделирования можно устранить, если принять, что величина поверхностной свободной энергии критического зародыша зависит от его размера, т.е. $\gamma=\gamma(R)$. По МД-данным о частоте зародышеобразования из уравнений $(5),(10)$, (13) рассчитана эффективная поверхностная свободная энергия $\gamma_{e}$ межфазной границы перегретый кристаллкритический зародыш. Результаты расчетов $\gamma_{e}$ для случая $e=0$ представлены на рис. 5 в зависимости от кривизны поверхности натяжения. Значение $\gamma_{e}$ при $e=0$ на $30-35 \%$ меньше, чем на плоской межфазной границе. Учет упругой энергии увеличивает это расхождение. При температурах $T^{*}=0.85,1.0$ отклонения составляют уже $50 \%$ и меняется характер зависимости $\gamma_{e}$ от $R$. Величина $\gamma_{e}(R)$ становится не убывающей, а возрастающей функцией кривизны.

При учете размерной зависимости поверхностной свободной энергии критического зародыша во всем исследованном диапазоне параметров состояния $W_{*} / k_{\mathrm{B}} T=13-22$. 


\section{5. Заключение}

При положительных и отрицательных давлениях исследована кинетика спонтанного плавления леннард-джонсовского ГЦК-кристалла. В молекулярнодинамических экспериментах рассчитана частота зародышеобразования, коэффициент диффузии зародышей, неравновесный фактор Зельдовича, размер критического зародыша. Расчеты проведены по четырем изотермам. Две изотермы имели температуру выше температуры тройной точки. У двух других температуры были близки к температуре тройной точки и конечной точки линии плавления - точки, в которой метастабильное продолжение линии плавления встречается со спинодалью растянутой жидкости (рис. 6). Температура предельного перегрева (предельного растяжения) определена в интервале приведенных частот зародышеобразования $10^{-9}-10^{-5}$. При $p=0$ и $J^{*}=10^{-7}$ предельный перегрев кристаллической фазы составил $1.2 T_{m}$, что хорошо согласуется с данными работы [5]. Спинодальному состоянию кристалла отвечает при этом температура перегрева $T_{s p}=1.29 T_{m}$. Изменение давления приводит к незначительному изменению относительной величины перегрева кристаллической фазы $\left(T_{n} / T_{m}=1.19-1.28\right)$. По мере приближения к температуре конечной точки линии плавления происходит сближение границы предельного перегрева и спинодали кристалла $[8,10,35]$.

Теория гомогенного зарождения жидкой фазы в перегретом кристалле строится на тех же принципах, что и теория кристаллизации, конденсации и кипения [1,11-13,15,36]. Мы сопоставили результаты молекулярно-динамического расчета основных характеристик процесса зародышеобразования с их значениями, полученными по формулам КТГЗ. Прямой МД-расчет величин $Z$ и $D_{*}$, а также радиуса критического зародыша $R_{*}$ показал, что определенный через них предэкспоненциальный множитель $J_{0}$ в уравнении (10) хорошо (в пределах 1-2 порядков) согласуется с его значением, рассчитанным по формулам КТГЗ (13), (14).

В твердом теле, в отличие от газа и жидкости, образование новой фазы связано с появлением упругой энергии внутренних напряжений. Мы определили величину упругой энергии для изотропной твердой фазы, находящейся под равномерным внешним давлением, считая зародыш сферическим. Вклад упругой энергии в работу образования критического зародыша уменьшается по мере понижения температуры (приближения к конечной точке кривой плавления) и роста величины перегрева (растяжения) кристаллической фазы. Рассчитанные прямым МД-моделированием размеры критических зародышей оказались несколько ниже полученных с помощью формулы КТГЗ (4). Причем, если при учете упругой энергии эти расхождения для $T^{*}=1.0$ достигали $50 \%$, то при $e=0$ они составили уже всего $10 \%$ и уменьшались по мере снижения температуры. Поскольку размер критического зародыша в существенной мере определяет работу его образования, а работа зародышеобразования

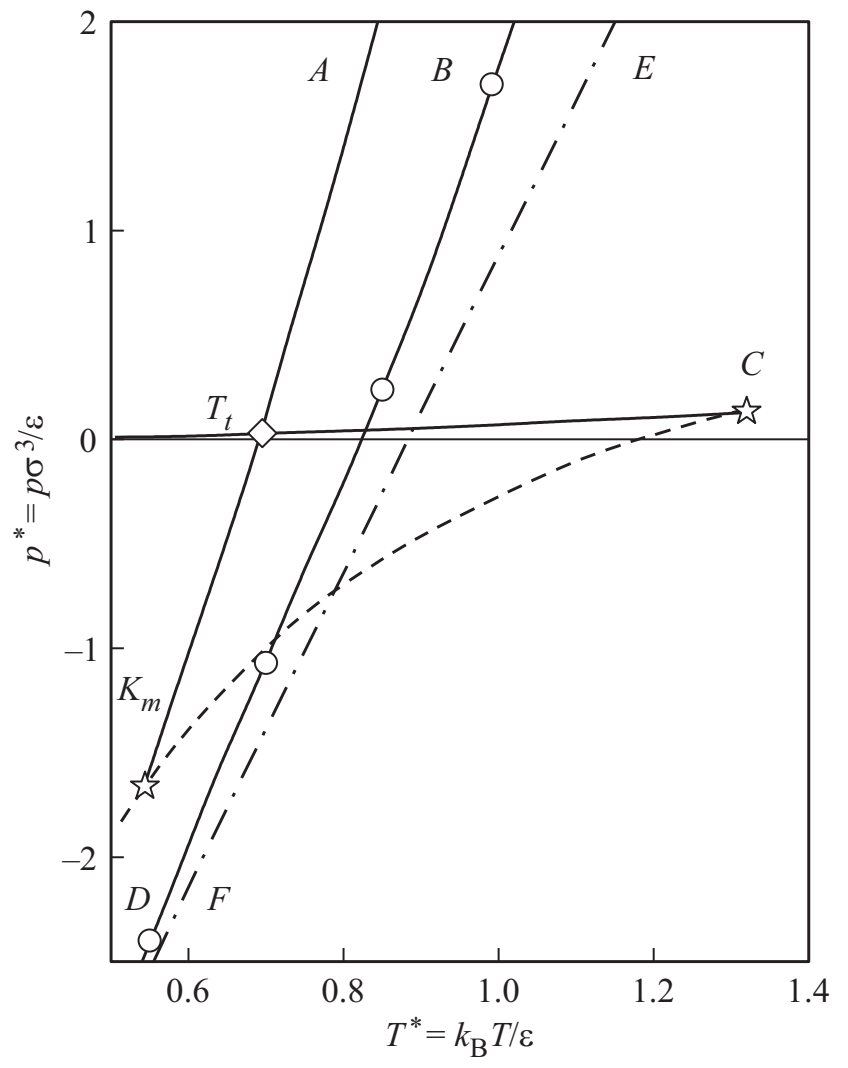

Рис. 6. Фазовая диаграмма леннард-джонсовской системы в координатах $p, T$. $A T_{t}$ - линия плавления и ее метастабильное продолжение $T_{t} K_{m} ; T_{t} C-$ линия насыщения; $K_{m} C-$ спинодаль перегретой жидкости; $B D$ - линия предельного перегрева, $J^{*}=10^{-7} ; E F-$ спинодаль перегретого кристалла; $T_{t}$ - тройная точка; $C-$ критическая точка; $K_{m}-$ конечная точка линии плавления.

входит в показатель экспоненты формулы для частоты зародышеобразования (10), то это приводит к значительным (до 70 порядков в $J$ при $e \neq 0$ ) отличиям в частоте зарождения новой фазы по данным МД-моделирования и КТГЗ. Столь значительные рассогласования не наблюдаются для конденсации пересыщенного пара [24,33] и кристаллизации переохлажденной жидкости [26,34]. В случае кавитации в растянутой леннард-джонсовской жидкости отличия в значениях $J$ МД-моделирования и КТГЗ достигали 20 порядков при $T^{*}=0.35$ и составили 9-10 порядков вблизи температуры тройной точки [32,37]. В работах [32,37] показано, что столь существенные рассогласования связаны с неучетом в КТГЗ зависимости поверхностной свободной энергии зародышей новой фазы от их размера.

Мы определили размерную зависимость эффективной поверхностной свободной энергии зародышей жидкой фазы в ЛД-кристалле из КТГЗ по данным МД-моделирования. Установлено, что если $e=0$, то величина $\gamma_{e}$ на изотерме убывает с уменьшением радиуса жидкой капли, причем отличия в значениях $\gamma_{e}$ и $\gamma$ составляют $30-35 \%$. 
Таким образом, проведенное исследование показало, что в отсутствие свободных поверхностей спонтанное плавление кристаллической фазы, по крайней мере качественно, может быть описано КТГЗ. Для более детального сопоставления КТГЗ и результатов МД-моделирования необходимо провести прямой расчет высоты активационного барьера и получить независимые данные о размерной зависимости поверхностной свободной энергии на границе кристалл-жидкая капля.

\section{Список литературы}

[1] И.М. Лифшиц, Л.С. Гулида. ДАН СССР 87, 3, 377 (1952).

[2] Г.И. Канель, В.Е. Фортов, С.В. Разоренов. УФН 177, 8, 809 (2007).

[3] С.И. Ашитков, П.С. Комаров, М.Б. Агранат, Г.И. Канель, В.Е. Фортов. Письма в ЖЭТФ 98, 7, 439 (2013).

[4] В.Г. Байдаков, А.Е. Галашев, В.П. Скрипов. ФТТ 2, 9, 2681 (1980).

[5] K. Lu, Y. Li. Phys. Rev. Lett. 80, 20, 4474 (1998).

[6] S.-N. Luo, T.J. Ahres, T. Cagin, A. Strachan, W.A. Goddard, D.C. Swift. Phys. Rev. B 68, 134206 (2003).

[7] J. Wang, J. Li, S. Yip, S. Phillpot, D. Wolf. Phys. Rev. B 52, 17, 12627 (1995).

[8] V.G. Baidakov, S.P. Protsenko. Phys. Rev. Lett. 95, 015701 (2005).

[9] S.-N. Luo, L. Zheng, A. Strachan, D.C. Swift. J. Chem. Phys. 126, 034505 (2007).

[10] А.Ю. Куксин, Г.Э. Норман, В.В. Стегайлов. ТВТ 45, 1, 43 (2007).

[11] В.И. Моторин. ФТТ 29, 4, 1227 (1987)

[12] E.A. Brener, S.V. Iordanskii, V.I. Marchenko. Phys. Rev. Lett. 82, 7, 1506 (1999).

[13] А.Л. Ройтбурд, Д.Е. Темкин. ФТТ 28, 3, 775 (1986).

[14] V.G. Baidakov, A.O. Tipeev. J. Chem. Phys. 143, 124501 (2015).

[15] Я.Б. Зельдович. ЖЭТФ 12, 11/12, 525 (1942).

[16] Е.М. Лифшиц, Л.П. Питаевский. Физическая кинетика. Наука, М. (1979). Т. 10. 528 с.

[17] Б.Я. Любов. Теория кристаллизации в больших объемах. Наука, М. (1975). 256 с.

[18] С. Глесстон, К. Лейдлер, Г. Эйринг. Теория абсолютных скоростей реакций. ИЛ, М. (1948). 293 с.

[19] В.П. Скрипов, В.П. Коверда. Спонтанная кристаллизация переохлажденных жидкостей. Наука, М. (1984). 232 с.

[20] А.В. Прохоров. ДАН СССР 239, 6, 1323 (1978).

[21] D. Frenkel, B. Smit. Understanding Molecular Simulations: From Algorithms to Applications. Academic Press , San Diego, San Francisco, N. Y., Boston, London, Sydney, Tokyo (1996). $443 \mathrm{p}$.

[22] L. Verlet. Phys. Rev. 159, 1, 98 (1967).

[23] В.П. Скрипов. Метастабильная жидкость. Наука, М. (1972). $312 \mathrm{c}$

[24] J. Wedekind, R. Strey, D. Reguera. J. Chem. Phys. 126, 134103 (2007).

[25] W. Lechner, C. Dellago. J. Chem. Phys. 129, 114707 (2008).

[26] V.G. Baidakov, A.O. Tipeev. J. Chem. Phys. 136, 174510 (2012).

[27] S. Auer, D. Frenkel. J. Chem. Phys. 120, 3015 (2004).
[28] V.G. Baidakov, S.P. Protsenko, A.O. Tipeev. J. Chem. Phys. 139, 224703 (2013).

[29] V.G. Baidakov, Z.R. Kozlova, S.P. Protsenko. Fluid Phase Equilib. 263, 55 (2008).

[30] В.Г. Байдаков, А.О. Типеев. ТВТ 56, 2, 193 (2018).

[31] Г.Ш. Болтачев, В.Г. Байдаков. ТВТ 41, 2, 314 (2003).

[32] V.G. Baidakov. J. Chem. Phys. 144, 074502 (2016).

[33] B.J. Block, S.K. Das, M. Oettel, P. Virnau, K. Binder. J. Chem. Phys. 133, 154702 (2010).

[34] V.G. Baidakov, A.O. Tipeev, K.S. Bobrov, G.V. Ionov. J. Chem. Phys. 132, 234505 (2010).

[35] A.Yu. Kuksin, G.E. Norman, V.V. Stegailov. Molecular Dynamics Modelling of Lifetime and Decay of Metastable Crystals Under Superheating or Stretching. in Computational Physics: Proceedings of the Joint Conference of ICCP6 and CCP2003. Rinton Press Inc., Beijing (2004). P. 126-129.

[36] V.I. Motorin, S.L. Musher. J. Chem. Phys. 91, 465 (1984).

[37] V.G. Baidakov, K.S. Bobrov. J. Chem. Phys. 140, 184506 (2014).

Редактор Д.В. Жуманов 